\title{
Triangular norms which are join-morphisms in 3-dimensional fuzzy set theory
}

\author{
Glad Deschrijver \\ Fuzziness and Uncertainty Modelling Research Unit, \\ Department of Applied Mathematics, Computer Science and Statistics, \\ Ghent University, Krijgslaan 281 (S9), B-9000 Gent, Belgium
}

\begin{abstract}
The $n$-dimensional fuzzy sets have been introduced as a generalization of interval-valued fuzzy sets, Atanassov's intuitionistic and interval-valued intuitionistic fuzzy sets. In this paper we investigate t-norms on 3-dimensional sets which are joinmorphisms. Under some additional conditions we show that they can be represented using a representation which generalizes a similar representation for t-norms in interval-valued fuzzy set theory.
\end{abstract}

Keywords: Triangular norm, join-morphism, interval-valued fuzzy set, Atanassov's intuitionistic fuzzy set, interval-valued intuitionistic fuzzy set, $n$-dimensional fuzzy set

\section{Introduction}

Zadeh [1] and Goguen [2] proposed the concepts of fuzzy sets and $L$-fuzzy sets in 1965 and 1967, respectively. Since then, several special $L$-fuzzy sets such as the interval-valued fuzzy set [3], the intuitionistic fuzzy set defined by Atanassov $[4,5]$ and the interval-valued fuzzy set [5] have been proposed. Interval-valued fuzzy set theory generalizes fuzzy set theory by providing for each element in the universe a closed subinterval of $[0,1]$ which approximates the real, but unknown, membership degree. In this way, it is not only possible to model vagueness, but also uncertainty about the membership degrees. Atanassov's intuitionistic fuzzy sets on the other hand provide for each element of the universe a degree of membership and a degree of non-membership, thus modelling the fact that the knowledge about the membership of an element in a set can be provided in a bipolar way: on the one hand there is information available which confirms membership, on the other hand there is information which infirms membership. A combination of these two theories is given by Atanassov's intervalvalued intuitionistic fuzzy sets: the membership degree and non-membership degree are both replaced by a closed subinterval of the unit interval which approximates the corresponding unknown degree.

Li et al. [6] and Shang et al. [7] introduced ndimensional fuzzy sets as a generalization for both interval-valued fuzzy sets and interval-valued intuitionistic fuzzy sets. In fact, interval-valued fuzzy sets are 2-dimensional fuzzy sets and interval-valued intuitionistic fuzzy sets can be seen as 4-dimensional fuzzy sets. The study of triangular norms and conorms, implication and negation functions, and in particular the study of t-norms satisfying the residuation principle, has been successful in fuzzy set theory (see e.g. $[8,9,10,11,12,13,14,15])$ and in interval-valued and Atanassov's intuitionistic fuzzy set theory (see e.g. $[16,17,18,19])$. In this paper we start the investigation of t-norms which are joinmorphisms in $n$-dimensional fuzzy sets. Given the complexity of this task, we will restrict our investigation in this paper to 3-dimensional fuzzy sets.

\section{Definitions}

Definition 1 [7] Let $n \in \mathbb{N} \backslash\{0\}$. We define the lattice $\mathcal{L}_{n}=\left(L_{n}, \leq_{n}\right)$ by

$$
\begin{aligned}
& L_{n}=\left\{\left(x_{1}, x_{2}, \ldots, x_{n}\right) \mid\left(x_{1}, \ldots, x_{n}\right) \in[0,1]^{n}\right. \\
& \left.\quad \text { and } x_{1} \leq x_{2} \leq \ldots \leq x_{n}\right\} \\
& \left(x_{1}, \ldots, x_{n}\right) \leq_{n}\left(y_{1}, \ldots, y_{n}\right) \\
& \quad \Longleftrightarrow(\forall i \in\{1, \ldots, n\})\left(x_{i} \leq y_{i}\right), \\
& \quad \text { for all }\left(x_{1}, \ldots, x_{n}\right),\left(y_{1}, \ldots, y_{n}\right) \text { in } L_{n} .
\end{aligned}
$$

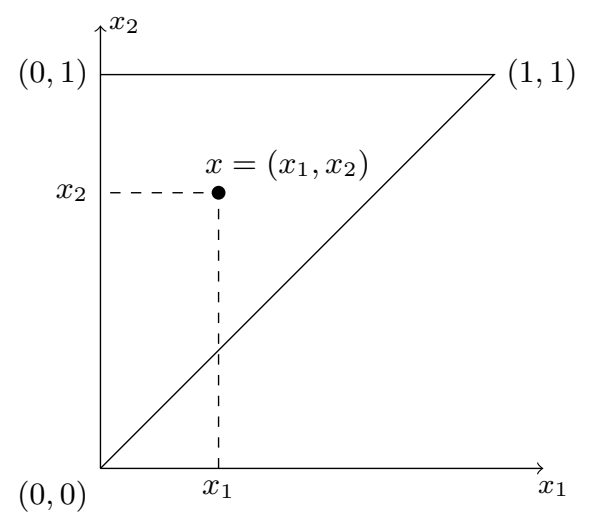

Figure 1: The lattice $\mathcal{L}_{2}$.

The lattices $\mathcal{L}_{2}$ and $\mathcal{L}_{3}$ are depicted in Figure 1 and Figure 2 respectively.

We denote the smallest and the largest element of $\mathcal{L}_{n}$ by $0_{\mathcal{L}_{n}}=(0, \ldots, 0)$ and $1_{\mathcal{L}_{n}}=(1, \ldots, 1)$ respectively. In the sequel, for any $x \in L_{n}$ and $i \in\{1, \ldots, n\}$, we will denote the $i$-th component 


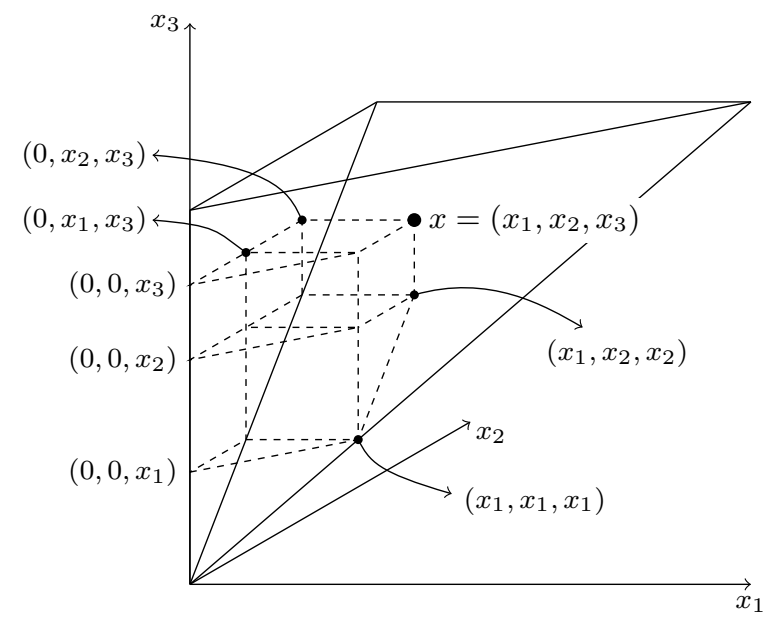

Figure 2: The lattice $\mathcal{L}_{3}$.

of $x$ by $x_{i}$, i.e. $x=\left(x_{1}, \ldots, x_{n}\right)$, or, equivalently, $\operatorname{pr}_{i} x=x_{i}$.

We define for all $\left\{i_{1}, i_{2}, \ldots, i_{k}\right\} \subseteq\{1, \ldots, n\}$ satisfying $i_{1}<i_{2}<\ldots<i_{k}$, the projection mapping $\operatorname{pr}_{i_{1}, i_{2}, \ldots, i_{k}}: L_{n} \rightarrow L_{k}:\left(x_{1}, \ldots, x_{n}\right) \mapsto$ $\left(x_{i_{1}}, \ldots, x_{i_{k}}\right)$, for all $\left(x_{1}, \ldots, x_{n}\right) \in L_{n}$.

Note that, for $x, y$ in $L_{n}, x<_{n} y$ is equivalent to " $x \leq_{n} y$ and $x \neq y$ ", i.e. $x_{i} \leq y_{i}$, for all $i \in\{1, \ldots, n\}$, and there exists at least one $i \in\{1, \ldots, n\}$ for which $x_{i}<y_{i}$. We define the subset $D_{n}$ of $L_{n}$ as $D_{n}=\left\{\left(x_{1}, x_{1}, \ldots, x_{1}\right) \mid x_{1} \in[0,1]\right\}$. We call the elements of $D_{n}$ the exact elements of $L_{n}$.

Note that for any non-empty subset $A$ of $L_{3}$ it holds that

$$
\begin{array}{r}
\sup A=\left(\sup \left\{x_{1} \mid\left(x_{1}, x_{2}, x_{3}\right) \in A\right\},\right. \\
\quad \sup \left\{x_{2} \mid\left(x_{1}, x_{2}, x_{3}\right) \in A\right\}, \\
\left.\quad \sup \left\{x_{3} \mid\left(x_{1}, x_{2}, x_{3}\right) \in A\right\}\right),
\end{array}
$$

and similarly for non-empty subsets of $L_{n}$, for any $n \in \mathbb{N} \backslash\{0\}$.

Definition 2 [7] Let $U$ be a set and $L_{n}^{U}=\{A \mid$ $A: U \rightarrow L_{n}$ is a mapping $\}$. Then each $A \in L_{n}^{U}$ is called an n-dimensional fuzzy set in $U$ and is denoted as

$$
A(u)=\left(A_{1}(u), A_{2}(u), \ldots, A_{n}(u)\right), \quad \forall u \in U
$$

Shang et al. [7] observed that $L_{1}^{U}$ is the set of Zadeh fuzzy sets on $U, L_{2}^{U}$ is isomorphic to the set of interval-valued fuzzy sets and to the set of Atanassov's intuitionistic fuzzy sets, and $L_{4}^{U}$ is isomorphic to the set of interval-valued intuitionistic fuzzy sets.

Definition 3 Let $n \in \mathbb{N} \backslash\{0\}$. A triangular norm (t-norm) on $\mathcal{L}_{n}$ is a commutative, associative mapping $\mathcal{T}:\left(L_{n}\right)^{2} \rightarrow L_{n}$ which is increasing in both places and for which $\mathcal{T}\left(1_{\mathcal{L}_{n}}, x\right)=x$, for all $x \in L_{n}$.
We say that a t-norm $\mathcal{T}$ on $\mathcal{L}_{n}$ satisfies the residuation principle if [10] for all $x, y$ and $z$ in $L_{n}$,

$$
\mathcal{T}(x, y) \leq_{n} z \Longleftrightarrow x \leq_{n} \mathcal{I}_{\mathcal{T}}(y, z),
$$

where for all $y$ and $z$ in $L_{n}$,

$$
\mathcal{I}_{\mathcal{T}}(y, z)=\sup \left\{\lambda \mid \lambda \in L_{n} \text { and } \mathcal{T}(\lambda, y) \leq_{n} z\right\} .
$$

The function $\mathcal{I}_{\mathcal{T}}$ is called the residuum of $\mathcal{T}$. The residuation principle is equivalent with the condition $[20,10,21]$ : for all $x \in L_{n}$ and $\emptyset \subset Z \subseteq L_{n}$,

$$
\mathcal{T}(x, \sup Z)=\sup _{z \in Z} \mathcal{T}(x, z) .
$$

We call a t-norm which satisfies the previous condition a sup-morphism. A weaker condition is: for all $x, y$ and $z$ in $L_{n}$,

$$
\mathcal{T}(x, \sup (y, z))=\sup (\mathcal{T}(x, y), \mathcal{T}(x, z)) .
$$

We call a t-norm which satisfies this condition a join-morphism.

\section{Triangular norms which are join-morphisms}

Proposition 1 [22, 17] Let $\mathcal{T}$ be a t-norm on $\mathcal{L}_{2}$ which is a join-morphism. Then there exists a $t$ norm $T$ on $([0,1], \leq)$ such that, for all $x$ and $y$ in $L_{2}$,

$$
\operatorname{pr}_{1} \mathcal{T}(x, y)=T\left(x_{1}, y_{1}\right) .
$$

In a similar way as the previous proposition, we can show the following.

Lemma 1 Let $\mathcal{T}$ be a t-norm on $\mathcal{L}_{n}$ which is a joinmorphism. Then there exists a $t$-norm $\mathcal{T}^{\prime}$ on $L_{n-1}$ which is a join-morphism such that, for all $x$ and $y$ in $L_{n}$,

$$
\begin{aligned}
& \operatorname{pr}_{1, \ldots, n-1} \mathcal{T}(x, y) \\
& =\mathcal{T}^{\prime}\left(\left(x_{1}, \ldots, x_{n-1}\right),\left(y_{1}, \ldots, y_{n-1}\right)\right) .
\end{aligned}
$$

The previous lemma is very important because it shows that in order to find the structure of a t-norm on $L_{n}$ which is a join-morphism, we can use for the first $n-1$ components the results for t-norms on $L_{n-1}$ and we only have to investigate the structure for the $n$-th component.

Theorem 1 [23, 17] Let $\mathcal{T}:\left(L_{2}\right)^{2} \rightarrow L_{2}$ be a mapping for which the mapping $g:[0,1] \rightarrow[0,1]$ defined by $g\left(y_{1}\right)=\operatorname{pr}_{2} \mathcal{T}\left((0,1),\left(y_{1}, y_{1}\right)\right)$, for all $y_{1} \in[0,1]$, satisfies $g=\mathrm{id}_{[0,1]}$. Then $\mathcal{T}$ is a t-norm on $\mathcal{L}_{2}$ which is a join-morphism and for which $\mathcal{T}\left(D_{2}, D_{2}\right) \subseteq D_{2}$ if and only if there exists an element $t_{2,1} \in[0,1]$ and a t-norm $T$ on $([0,1], \leq)$ such that $T$ is a joinmorphism and for all $x$ and $y$ in $L_{2}$,

$$
\begin{gathered}
\mathcal{T}(x, y)=\left(T\left(x_{1}, y_{1}\right), \sup \left(T\left(t_{2,1}, T\left(x_{2}, y_{2}\right)\right),\right.\right. \\
\left.\left.T\left(x_{1}, y_{2}\right), T\left(x_{2}, y_{1}\right)\right)\right) .
\end{gathered}
$$


Combining Lemma 1 and Theorem 1, we obtain the following result.

Lemma 2 Let $\mathcal{T}:\left(L_{3}\right)^{2} \rightarrow L_{3}$ be a $t$-norm on $\mathcal{L}_{3}$ for which the mapping $g:[0,1] \rightarrow[0,1]$ defined by $g\left(y_{1}\right)=\operatorname{pr}_{2} \mathcal{T}\left((0,1,1),\left(y_{1}, y_{1}, y_{1}\right)\right)$, for all $y_{1} \in[0,1]$, satisfies $g=\mathrm{id}_{[0,1]}$. If $\mathcal{T}$ is a joinmorphism and satisfies $\mathcal{T}\left(D_{3}, D_{3}\right) \subseteq D_{3}$, then there exists an element $t_{2,1} \in[0,1]$ and a t-norm $T$ on $([0,1], \leq)$ such that $T$ is a join-morphism and for all $x$ and $y$ in $L_{3}$,

$$
\begin{gathered}
\operatorname{pr}_{1,2} \mathcal{T}(x, y)=\left(T\left(x_{1}, y_{1}\right), \sup \left(T\left(t_{2,1}, T\left(x_{2}, y_{2}\right)\right)\right.\right. \\
\left.\left.T\left(x_{1}, y_{2}\right), T\left(x_{2}, y_{1}\right)\right)\right)
\end{gathered}
$$

Lemma 2 determines the first two components of a t-norm on $\mathcal{L}_{3}$ which is a join-morphism and which satisfies some boundary conditions. In what follows we establish a representation of the third component. First we need some lemmas.

Lemma 3 Let $\mathcal{T}$ be a t-norm on $\mathcal{L}_{3}$ which is a joinmorphism and which satisfies $\mathcal{T}\left(D_{3}, D_{3}\right) \subseteq D_{3}$. Define the mappings $f_{1}, f_{2}, g_{1}, g_{2}, h, T:[0,1]^{2} \rightarrow[0,1]$ by, for all $x_{1}, x_{2}, x_{3}, y_{1}, y_{2}, y_{3}$ in $[0,1]$,

$$
\begin{aligned}
f_{1}\left(x_{3}, y_{3}\right) & =\operatorname{pr}_{3} \mathcal{T}\left(\left(0,0, x_{3}\right),\left(0,0, y_{3}\right)\right), \\
f_{2}\left(x_{2}, y_{2}\right) & =\operatorname{pr}_{3} \mathcal{T}\left(\left(0, x_{2}, x_{2}\right),\left(0, y_{2}, y_{2}\right)\right), \\
g_{1}\left(x_{1}, y_{3}\right) & =\operatorname{pr}_{3} \mathcal{T}\left(\left(x_{1}, x_{1}, x_{1}\right),\left(0,0, y_{3}\right)\right), \\
g_{2}\left(x_{1}, y_{2}\right) & =\operatorname{pr}_{3} \mathcal{T}\left(\left(x_{1}, x_{1}, x_{1}\right),\left(0, y_{2}, y_{2}\right)\right), \\
h\left(x_{2}, y_{3}\right) & =\operatorname{pr}_{3} \mathcal{T}\left(\left(0, x_{2}, x_{2}\right),\left(0,0, y_{3}\right)\right), \\
T\left(x_{1}, y_{1}\right) & =\operatorname{pr}_{3} \mathcal{T}\left(\left(x_{1}, x_{1}, x_{1}\right),\left(y_{1}, y_{1}, y_{1}\right)\right) .
\end{aligned}
$$

Then, for all $x$ and $y$ in $L_{3}$,

$$
\begin{aligned}
\operatorname{pr}_{3} \mathcal{T}(x, y)=\sup \left(f_{1}\left(x_{3}, y_{3}\right), f_{2}\left(x_{2}, y_{2}\right), g_{1}\left(x_{1}, y_{3}\right),\right. & \\
& g_{1}\left(y_{1}, x_{3}\right), g_{2}\left(x_{1}, y_{2}\right), g_{2}\left(y_{1}, x_{2}\right), \\
& \left.h\left(x_{2}, y_{3}\right), h\left(y_{2}, x_{3}\right), T\left(x_{1}, y_{1}\right)\right) .
\end{aligned}
$$

Proof. This follows easily from the fact that $\mathcal{T}$ is a t-norm which is a join-morphism and which satisfies $\mathcal{T}\left(D_{3}, D_{3}\right) \subseteq D_{3}$ and from the fact that, for all $x \in$ $L_{3}$,

$$
x=\sup \left(\left(x_{1}, x_{1}, x_{1}\right),\left(0, x_{2}, x_{2}\right),\left(0,0, x_{3}\right)\right),
$$

and similarly for $y \in L_{3}$.

Lemma 4 Let $\mathcal{T}$ be a t-norm on $\mathcal{L}_{3}$ which is a joinmorphism and which satisfies $\mathcal{T}\left(D_{3}, D_{3}\right) \subseteq D_{3}$. Define the mappings $f_{1}, f_{2}, g_{1}, g_{2}, h, T:[0,1]^{2} \rightarrow[0,1]$ by (1). Then, for all $x_{1}$ and $y_{1}$ in $[0,1]$,

$$
\begin{aligned}
f_{1}\left(x_{1}, y_{1}\right) & \leq h\left(x_{1}, y_{1}\right) \leq g_{1}\left(x_{1}, y_{1}\right) \leq g_{2}\left(x_{1}, y_{1}\right) \\
h\left(x_{1}, y_{1}\right) & \leq f_{2}\left(x_{1}, y_{1}\right) \leq g_{2}\left(x_{1}, y_{1}\right) \leq T\left(x_{1}, y_{1}\right)
\end{aligned}
$$

Proof. This follows immediately from Lemma 3 and the fact that $\mathcal{T}$ is increasing.
Lemma 5 Let $\mathcal{T}$ be a t-norm on $\mathcal{L}_{3}$ which is a joinmorphism and which satisfies $\mathcal{T}\left(D_{3}, D_{3}\right) \subseteq D_{3}$. Define the mappings $f_{1}, f_{2}, g_{1}, g_{2}, h, T:[0,1]^{2} \rightarrow[0,1]$ by (1). Then, for all $x_{1}, x_{2}, x_{3}, y_{1}, y_{2}, y_{3}, z_{1}, z_{2}$ and $z_{3}$ in $[0,1$,

$$
\begin{aligned}
f_{1}\left(x_{3}, h\left(z_{2}, y_{3}\right)\right) & =h\left(z_{2}, f_{1}\left(x_{3}, y_{3}\right)\right), \\
h\left(y_{2}, h\left(z_{2}, x_{3}\right)\right) & =h\left(z_{2}, h\left(y_{2}, x_{3}\right)\right), \\
f_{1}\left(x_{3}, g_{1}\left(z_{1}, y_{3}\right)\right) & =g_{1}\left(z_{1}, f_{1}\left(x_{3}, y_{3}\right)\right), \\
f_{1}\left(x_{3}, g_{1}\left(z_{1}, y_{3}\right)\right) & =f_{1}\left(y_{3}, g_{1}\left(z_{1}, x_{3}\right)\right), \\
g_{1}\left(y_{1}, g_{1}\left(z_{1}, x_{3}\right)\right) & =g_{1}\left(z_{1}, g_{1}\left(y_{1}, x_{3}\right)\right), \\
g_{1}\left(y_{1}, g_{1}\left(z_{1}, x_{3}\right)\right) & =g_{1}\left(T\left(y_{1}, z_{1}\right), x_{3}\right) .
\end{aligned}
$$

Proof. In the following calculations we use the fact that $\operatorname{pr}_{i} \mathcal{T}(x, y)=0$ as soon as $\min \left(x_{i}, y_{i}\right)=0$, for all $x$ and $y$ in $L_{3}$ and for all $i \in\{1,2\}$.

The first equality can be shown as follows:

$$
\begin{aligned}
& f_{1}\left(x_{3}, h\left(z_{2}, y_{3}\right)\right) \\
& =\operatorname{pr}_{3} \mathcal{T}\left(\left(0,0, x_{3}\right),\left(0,0, h\left(z_{2}, y_{3}\right)\right)\right) \\
& =\operatorname{pr}_{3} \mathcal{T}\left(\left(0,0, x_{3}\right), \mathcal{T}\left(\left(0,0, y_{3}\right),\left(0, z_{2}, z_{2}\right)\right)\right) \\
& =\operatorname{pr}_{3} \mathcal{T}\left(\mathcal{T}\left(\left(0,0, x_{3}\right),\left(0,0, y_{3}\right)\right),\left(0, z_{2}, z_{2}\right)\right) \\
& =\operatorname{pr}_{3} \mathcal{T}\left(\left(0,0, f_{1}\left(x_{3}, y_{3}\right)\right),\left(0, z_{2}, z_{2}\right)\right) \\
& =h\left(z_{2}, f_{1}\left(x_{3}, y_{3}\right)\right) .
\end{aligned}
$$

We prove the second equality as follows:

$$
\begin{aligned}
& h\left(y_{2}, h\left(z_{2}, x_{3}\right)\right) \\
& =\operatorname{pr}_{3} \mathcal{T}\left(\left(0, y_{2}, y_{2}\right),\left(0,0, h\left(z_{2}, x_{3}\right)\right)\right) \\
& =\operatorname{pr}_{3} \mathcal{T}\left(\left(0, y_{2}, y_{2}\right), \mathcal{T}\left(\left(0,0, x_{3}\right),\left(0, z_{2}, z_{2}\right)\right)\right) \\
& =\operatorname{pr}_{3} \mathcal{T}\left(\mathcal{T}\left(\left(0, y_{2}, y_{2}\right),\left(0,0, x_{3}\right)\right),\left(0, z_{2}, z_{2}\right)\right) \\
& =\operatorname{pr}_{3} \mathcal{T}\left(\left(0,0, h\left(y_{2}, x_{3}\right)\right),\left(0, z_{2}, z_{2}\right)\right) \\
& =h\left(z_{2}, h\left(y_{2}, x_{3}\right)\right) .
\end{aligned}
$$

The third formula is proven as follows:

$$
\begin{aligned}
& f_{1}\left(x_{3}, g_{1}\left(z_{1}, y_{3}\right)\right) \\
& =\operatorname{pr}_{3} \mathcal{T}\left(\left(0,0, x_{3}\right),\left(0,0, g_{1}\left(z_{1}, y_{3}\right)\right)\right) \\
& =\operatorname{pr}_{3} \mathcal{T}\left(\left(0,0, x_{3}\right), \mathcal{T}\left(\left(0,0, y_{3}\right),\left(z_{1}, z_{1}, z_{1}\right)\right)\right) \\
& =\operatorname{pr}_{3} \mathcal{T}\left(\mathcal{T}\left(\left(0,0, x_{3}\right),\left(0,0, y_{3}\right)\right),\left(z_{1}, z_{1}, z_{1}\right)\right) \\
& =\operatorname{pr}_{3} \mathcal{T}\left(\left(0,0, f_{1}\left(x_{3}, y_{3}\right)\right),\left(z_{1}, z_{1}, z_{1}\right)\right) \\
& =g_{1}\left(z_{1}, f_{1}\left(x_{3}, y_{3}\right)\right),
\end{aligned}
$$

and

$$
\begin{aligned}
& f_{1}\left(x_{3}, g_{1}\left(z_{1}, y_{3}\right)\right) \\
& =\operatorname{pr}_{3} \mathcal{T}\left(\left(0,0, x_{3}\right),\left(0,0, g_{1}\left(z_{1}, y_{3}\right)\right)\right) \\
& =\operatorname{pr}_{3} \mathcal{T}\left(\left(0,0, x_{3}\right), \mathcal{T}\left(\left(z_{1}, z_{1}, z_{1}\right),\left(0,0, y_{3}\right)\right)\right) \\
& =\operatorname{pr}_{3} \mathcal{T}\left(\mathcal{T}\left(\left(0,0, x_{3}\right),\left(z_{1}, z_{1}, z_{1}\right)\right),\left(0,0, y_{3}\right)\right) \\
& =\operatorname{pr}_{3} \mathcal{T}\left(\left(0,0, g_{1}\left(z_{1}, x_{3}\right)\right),\left(0,0, y_{3}\right)\right) \\
& =f_{1}\left(y_{3}, g_{1}\left(z_{1}, x_{3}\right)\right) .
\end{aligned}
$$


We show the fourth formula as follows:

$$
\begin{aligned}
& g_{1}\left(y_{1}, g_{1}\left(z_{1}, x_{3}\right)\right) \\
& =\operatorname{pr}_{3} \mathcal{T}\left(\left(y_{1}, y_{1}, y_{1}\right),\left(0,0, g_{1}\left(z_{1}, x_{3}\right)\right)\right) \\
& =\operatorname{pr}_{3} \mathcal{T}\left(\left(y_{1}, y_{1}, y_{1}\right), \mathcal{T}\left(\left(0,0, x_{3}\right),\left(z_{1}, z_{1}, z_{1}\right)\right)\right) \\
& =\operatorname{pr}_{3} \mathcal{T}\left(\mathcal{T}\left(\left(y_{1}, y_{1}, y_{1}\right),\left(0,0, x_{3}\right)\right),\left(z_{1}, z_{1}, z_{1}\right)\right) \\
& =\operatorname{pr}_{3} \mathcal{T}\left(\left(0,0, g_{1}\left(y_{1}, x_{3}\right)\right),\left(z_{1}, z_{1}, z_{1}\right)\right) \\
& =g_{1}\left(z_{1}, g_{1}\left(y_{1}, x_{3}\right)\right),
\end{aligned}
$$

and

$$
\begin{aligned}
& g_{1}\left(y_{1}, g_{1}\left(z_{1}, x_{3}\right)\right) \\
& =\operatorname{pr}_{3} \mathcal{T}\left(\left(y_{1}, y_{1}, y_{1}\right),\left(0,0, g_{1}\left(z_{1}, x_{3}\right)\right)\right) \\
& =\operatorname{pr}_{3} \mathcal{T}\left(\left(y_{1}, y_{1}, y_{1}\right), \mathcal{T}\left(\left(z_{1}, z_{1}, z_{1}\right),\left(0,0, x_{3}\right)\right)\right) \\
& =\operatorname{pr}_{3} \mathcal{T}\left(\mathcal{T}\left(\left(y_{1}, y_{1}, y_{1}\right),\left(z_{1}, z_{1}, z_{1}\right)\right),\left(0,0, x_{3}\right)\right) \\
& =\operatorname{pr}_{3} \mathcal{T}\left(\left(T\left(y_{1}, z_{1}\right), T\left(y_{1}, z_{1}\right), T\left(y_{1}, z_{1}\right)\right),\left(0,0, x_{3}\right)\right) \\
& =g_{1}\left(T\left(y_{1}, z_{1}\right), x_{3}\right)
\end{aligned}
$$

Lemma 6 Let $\mathcal{T}$ be a t-norm on $\mathcal{L}_{3}$ which is a joinmorphism and which satisfies $\mathcal{T}\left(D_{3}, D_{3}\right) \subseteq D_{3}$. Define the mappings $f_{1}, f_{2}, g_{1}, g_{2}, h, T:[0,1]^{2} \rightarrow[0,1]$ by (1). Assume that the mapping $g:[0,1] \rightarrow[0,1]$ defined by $g\left(y_{1}\right)=\operatorname{pr}_{2} \mathcal{T}\left((0,1,1),\left(y_{1}, y_{1}, y_{1}\right)\right)$, for all $y_{1} \in[0,1]$, satisfies $g=\mathrm{id}_{[0,1]}$. Then, for all $x_{1}$, $x_{2}, x_{3}, y_{1}, y_{2}, y_{3}, z_{1}, z_{2}$ and $z_{3}$ in $[0,1$,

$$
\begin{aligned}
g_{2} & =T \\
f_{2}\left(x_{2}, T\left(y_{2}, z_{1}\right)\right)= & f_{2}\left(y_{2}, T\left(x_{2}, z_{1}\right)\right), \\
f_{2}\left(x_{2}, T\left(y_{2}, z_{1}\right)\right)= & \sup \left(g_{2}\left(z_{1}, T\left(t_{2,1}, T\left(x_{2}, y_{2}\right)\right)\right),\right. \\
& \left.g_{1}\left(z_{1}, f_{2}\left(x_{2}, y_{2}\right)\right)\right), \\
g_{1}\left(z_{1}, h\left(y_{2}, x_{3}\right)\right)= & h\left(y_{2}, g_{1}\left(z_{1}, x_{3}\right)\right), \\
g_{1}\left(z_{1}, h\left(y_{2}, x_{3}\right)\right)= & h\left(T\left(z_{1}, y_{2}\right), x_{3}\right) .
\end{aligned}
$$

Proof. We first show that $g_{2}=T$. For any $x_{2}$ and $y_{1}$ in $[0,1]$ we find:

$\mathcal{T}\left(\left(0, x_{2}, x_{2}\right),\left(y_{1}, y_{1}, y_{1}\right)\right)=\left(0, T\left(y_{1}, x_{2}\right), g_{2}\left(y_{1}, x_{2}\right)\right)$.

Since the range of $\mathcal{T}$ is a subset of $L_{3}$, it follows that $T\left(y_{1}, x_{2}\right) \leq g_{2}\left(y_{1}, x_{2}\right)$. Combining this with Lemma 4 leads to the equality.

We show the second formula:

$$
\begin{aligned}
& f_{2}\left(x_{2}, T\left(y_{2}, z_{1}\right)\right) \\
& =\operatorname{pr}_{3} \mathcal{T}\left(\left(0, x_{2}, x_{2}\right),\left(0, T\left(y_{2}, z_{1}\right), T\left(y_{2}, z_{1}\right)\right)\right) \\
& =\operatorname{pr}_{3} \mathcal{T}\left(\left(0, x_{2}, x_{2}\right), \mathcal{T}\left(\left(z_{1}, z_{1}, z_{1}\right),\left(0, y_{2}, y_{2}\right)\right)\right) \\
& =\operatorname{pr}_{3} \mathcal{T}\left(\mathcal{T}\left(\left(0, x_{2}, x_{2}\right),\left(z_{1}, z_{1}, z_{1}\right)\right),\left(0, y_{2}, y_{2}\right)\right) \\
& =\operatorname{pr}_{3} \mathcal{T}\left(\left(0, T\left(x_{2}, z_{1}\right), T\left(x_{2}, z_{1}\right)\right),\left(0, y_{2}, y_{2}\right)\right) \\
& =f_{2}\left(y_{2}, T\left(x_{2}, z_{1}\right)\right),
\end{aligned}
$$

and

$$
\begin{aligned}
f_{2}\left(x_{2}, T\left(y_{2}, z_{1}\right)\right) \\
=\operatorname{pr}_{3} \mathcal{T}\left(\left(0, x_{2}, x_{2}\right),\left(0, T\left(y_{2}, z_{1}\right), T\left(y_{2}, z_{1}\right)\right)\right) \\
=\operatorname{pr}_{3} \mathcal{T}\left(\left(0, x_{2}, x_{2}\right), \mathcal{T}\left(\left(0, y_{2}, y_{2}\right),\left(z_{1}, z_{1}, z_{1}\right)\right)\right) \\
=\operatorname{pr}_{3} \mathcal{T}\left(\mathcal{T}\left(\left(0, x_{2}, x_{2}\right),\left(0, y_{2}, y_{2}\right)\right),\left(z_{1}, z_{1}, z_{1}\right)\right) \\
=\operatorname{pr}_{3} \mathcal{T}\left(\left(0, T\left(t_{2,1}, T\left(x_{2}, y_{2}\right)\right), f_{2}\left(x_{2}, y_{2}\right)\right),\right. \\
\left.\quad\left(z_{1}, z_{1}, z_{1}\right)\right)
\end{aligned}
$$

$$
\begin{aligned}
= & \operatorname{pr}_{3} \sup \left(\mathcal { T } \left(\left(0, T\left(t_{2,1}, T\left(x_{2}, y_{2}\right)\right),\right.\right.\right. \\
& \left.\left.\quad T\left(t_{2,1}, T\left(x_{2}, y_{2}\right)\right)\right),\left(z_{1}, z_{1}, z_{1}\right)\right), \\
& \left.\mathcal{T}\left(\left(0,0, f_{2}\left(x_{2}, y_{2}\right)\right),\left(z_{1}, z_{1}, z_{1}\right)\right)\right) \\
= & \sup \left(g_{2}\left(z_{1}, T\left(t_{2,1}, T\left(x_{2}, y_{2}\right)\right)\right), g_{1}\left(z_{1}, f_{2}\left(x_{2}, y_{2}\right)\right)\right) .
\end{aligned}
$$

The equalities in the third formula are shown as follows:

$$
\begin{aligned}
& g_{1}\left(z_{1}, h\left(y_{2}, x_{3}\right)\right) \\
& =\operatorname{pr}_{3} \mathcal{T}\left(\left(z_{1}, z_{1}, z_{1}\right),\left(0,0, h\left(y_{2}, x_{3}\right)\right)\right) \\
& =\operatorname{pr}_{3} \mathcal{T}\left(\left(z_{1}, z_{1}, z_{1}\right), \mathcal{T}\left(\left(0,0, x_{3}\right),\left(0, y_{2}, y_{2}\right)\right)\right) \\
& =\operatorname{pr}_{3} \mathcal{T}\left(\mathcal{T}\left(\left(z_{1}, z_{1}, z_{1}\right),\left(0,0, x_{3}\right)\right),\left(0, y_{2}, y_{2}\right)\right) \\
& =\operatorname{pr}_{3} \mathcal{T}\left(\left(0,0, g_{1}\left(z_{1}, x_{3}\right)\right),\left(0, y_{2}, y_{2}\right)\right) \\
& =h\left(y_{2}, g_{1}\left(z_{1}, x_{3}\right)\right),
\end{aligned}
$$

and

$$
\begin{aligned}
& g_{1}\left(z_{1}, h\left(y_{2}, x_{3}\right)\right) \\
& =\operatorname{pr}_{3} \mathcal{T}\left(\left(z_{1}, z_{1}, z_{1}\right),\left(0,0, h\left(y_{2}, x_{3}\right)\right)\right) \\
& =\operatorname{pr}_{3} \mathcal{T}\left(\left(z_{1}, z_{1}, z_{1}\right), \mathcal{T}\left(\left(0, y_{2}, y_{2}\right),\left(0,0, x_{3}\right)\right)\right) \\
& =\operatorname{pr}_{3} \mathcal{T}\left(\mathcal{T}\left(\left(z_{1}, z_{1}, z_{1}\right),\left(0, y_{2}, y_{2}\right)\right),\left(0,0, x_{3}\right)\right) \\
& =\operatorname{pr}_{3} \mathcal{T}\left(\left(0, T\left(z_{1}, y_{2}\right), T\left(z_{1}, y_{2}\right)\right),\left(0,0, x_{3}\right)\right) \\
& =h\left(T\left(z_{1}, y_{2}\right), x_{3}\right) .
\end{aligned}
$$

Lemma 7 Let $\mathcal{T}$ be a t-norm on $\mathcal{L}_{3}$ which is a joinmorphism and which satisfies $\mathcal{T}\left(D_{3}, D_{3}\right) \subseteq D_{3}$. Define the mappings $f_{1}, f_{2}, g_{1}, g_{2}, h, T:[0,1]^{2} \rightarrow[0,1]$ by (1). Assume that the mapping $g:[0,1] \rightarrow[0,1]$ defined by $g\left(y_{1}\right)=\operatorname{pr}_{2} \mathcal{T}\left((0,1,1),\left(y_{1}, y_{1}, y_{1}\right)\right)$, for all $y_{1} \in[0,1]$, satisfies $g=\mathrm{id}_{[0,1]}$. Assume furthermore that $g_{1}\left(x_{1}, 1\right)=x_{1}$, for all $x_{1} \in[0,1]$. Then, for all $x_{1}, x_{2}, x_{3}, y_{1}, y_{2}, y_{3}, z_{1}, z_{2}$ and $z_{3}$ in $[0,1$,

$$
\begin{aligned}
g_{1} & =T, \\
f_{1}\left(y_{3}, z_{1}\right) & =T\left(T\left(z_{1}, y_{3}\right), f_{1}(1,1)\right), \\
f_{2}\left(x_{2}, z_{1}\right) & =T\left(T\left(z_{1}, x_{2}\right), f_{2}(1,1)\right), \\
h\left(z_{1}, x_{3}\right) & =T\left(T\left(z_{1}, x_{3}\right), h(1,1)\right) .
\end{aligned}
$$

Proof. From Lemma 5 we know that for all $y_{1}$ and $z_{1}$ in $[0,1]$,

$$
g_{1}\left(y_{1}, g_{1}\left(z_{1}, 1\right)\right)=g_{1}\left(T\left(y_{1}, z_{1}\right), 1\right),
$$

which together with the given condition on $g_{1}$ yields that $g_{1}\left(y_{1}, z_{1}\right)=T\left(y_{1}, z_{1}\right)$.

For any $y_{3}$ and $z_{1}$ in $[0,1]$, we know from Lemma 5 and the previous result that

$$
\begin{aligned}
f_{1}\left(1, T\left(z_{1}, y_{3}\right)\right) & =f_{1}\left(1, g_{1}\left(z_{1}, y_{3}\right)\right) \\
& =f_{1}\left(y_{3}, g_{1}\left(z_{1}, x_{3}\right)\right) \\
& =f_{1}\left(y_{3}, z_{1}\right)
\end{aligned}
$$

and

$$
\begin{aligned}
f_{1}\left(1, z_{1}\right) & =f_{1}\left(1, g_{1}\left(z_{1}, 1\right)\right) \\
& =g_{1}\left(z_{1}, f_{1}(1,1)\right) \\
& =T\left(z_{1}, f_{1}(1,1)\right)
\end{aligned}
$$


from which we conclude that

$$
f_{1}\left(y_{3}, z_{1}\right)=T\left(T\left(z_{1}, y_{3}\right), f_{1}(1,1)\right) \text {. }
$$

For any $x_{2}$ and $z_{1}$ in $[0,1]$, we know from Lemma 6 and the previous result that

$$
f_{2}\left(x_{2}, z_{1}\right)=f_{2}\left(x_{2}, T\left(1, z_{1}\right)\right)=f_{2}\left(1, T\left(x_{2}, z_{1}\right)\right),
$$

and

$$
\begin{aligned}
& f_{2}\left(1, z_{1}\right) \\
& =f_{2}\left(1, T\left(1, z_{1}\right)\right) \\
& =\sup \left(g_{2}\left(z_{1}, T\left(t_{2,1}, T(1,1)\right)\right), g_{1}\left(z_{1}, f_{2}(1,1)\right)\right), \\
& =\sup \left(T\left(z_{1}, t_{2,1}\right), T\left(z_{1}, f_{2}(1,1)\right)\right) .
\end{aligned}
$$

Let $z_{1}=1$ in the above calculation, then we obtain $f_{2}(1,1)=\sup \left(t_{2,1}, f_{2}(1,1)\right)$. So from (2) it follows that

$$
f_{2}\left(1, z_{1}\right)=T\left(z_{1}, f_{2}(1,1)\right) .
$$

We conclude that

$$
f_{2}\left(x_{2}, z_{1}\right)=T\left(T\left(z_{1}, x_{2}\right), f_{2}(1,1)\right) .
$$

From Lemma 6 it follows for all $x_{3}$ and $z_{1}$ in $[0,1]$ that

$$
\begin{aligned}
h\left(z_{1}, x_{3}\right) & =h\left(T\left(z_{1}, 1\right), x_{3}\right) \\
& =g_{1}\left(z_{1}, h\left(1, x_{3}\right)\right) \\
& =h\left(1, g_{1}\left(z_{1}, x_{3}\right)\right) \\
& =h\left(1, T\left(z_{1}, x_{3}\right)\right),
\end{aligned}
$$

and

$$
\begin{aligned}
T\left(z_{1}, h(1,1)\right) & =g_{1}\left(z_{1}, h(1,1)\right) \\
& =h\left(1, g_{1}\left(z_{1}, 1\right)\right) \\
& =h\left(1, z_{1}\right) .
\end{aligned}
$$

So

$$
h\left(z_{1}, x_{3}\right)=T\left(T\left(z_{1}, x_{3}\right), h(1,1)\right) .
$$

Lemma 8 Let $\mathcal{T}$ be a t-norm on $\mathcal{L}_{3}$ which is a joinmorphism and which satisfies $\mathcal{T}\left(D_{3}, D_{3}\right) \subseteq D_{3}$. Define the mappings $f_{1}, f_{2}, g_{1}, g_{2}, h, T:[0,1]^{2} \rightarrow[0,1]$ by (1). Assume that the mapping $g:[0,1] \rightarrow[0,1]$ defined by $g\left(y_{1}\right)=\operatorname{pr}_{2} \mathcal{T}\left((0,1,1),\left(y_{1}, y_{1}, y_{1}\right)\right)$, for all $y_{1} \in[0,1]$, satisfies $g=\mathrm{id}_{[0,1]}$. Assume furthermore that $g_{1}\left(x_{1}, 1\right)=x_{1}$, for all $x_{1} \in[0,1]$. Let

$$
\begin{aligned}
& t_{2,1}=\operatorname{pr}_{2} \mathcal{T}((0,1,1),(0,1,1)), \\
& t_{3,1}=f_{1}(1,1)=\operatorname{pr}_{3} \mathcal{T}((0,0,1),(0,0,1)), \\
& t_{3,2}=h(1,1)=\operatorname{pr}_{3} \mathcal{T}((0,0,1),(0,1,1)), \\
& t_{3,3}=f_{2}(1,1)=\operatorname{pr}_{3} \mathcal{T}((0,1,1),(0,1,1)) .
\end{aligned}
$$

Then

$$
T\left(t_{3,1}, t_{3,2}\right)=\sup \left(T\left(t_{3,1}, t_{3,3}\right), T\left(t_{3,2}, t_{2,1}\right)\right) .
$$

Proof. For $x=(0,1,1), y=(0,1,1)$ and $z=$ $(0,0,1)$ we find:

$$
\begin{aligned}
\operatorname{pr}_{3} \mathcal{T}(x, \mathcal{T}(y, z)) & =\operatorname{pr}_{3} \mathcal{T}((0,1,1),(0,0, h(1,1))) \\
& =h(1, h(1,1)),
\end{aligned}
$$

which by Lemma 7 is equal to $T(h(1,1), h(1,1))=$ $T\left(t_{3,2}, t_{3,2}\right)$.

On the other hand,

$$
\begin{aligned}
\operatorname{pr}_{3} \mathcal{T}(\mathcal{T}(x, y), z) \\
=\operatorname{pr}_{3} \mathcal{T}\left(\left(0, t_{2,1}, t_{3,3}\right),(0,0,1)\right) \\
=\operatorname{pr}_{3} \sup \left(\mathcal{T}\left(\left(0, t_{2,1}, t_{2,1}\right),(0,0,1)\right),\right. \\
\left.\quad \mathcal{T}\left(\left(0,0, t_{3,3}\right),(0,0,1)\right)\right) \\
=\sup \left(h\left(t_{2,1}, 1\right), f_{1}\left(t_{3,3}, 1\right)\right),
\end{aligned}
$$

which by Lemma 7 is equal to $\sup \left(T\left(t_{2,1}, h(1,1)\right)\right.$, $\left.T\left(t_{3,3}, f_{1}(1,1)\right)\right)=\sup \left(T\left(t_{2,1}, t_{3,2}\right), T\left(t_{3,3}, t_{3,1}\right)\right)$.

From Theorem 1, Lemma 3, Lemma 6, Lemma 7 and Lemma 8 it easily follows that the following representation holds.

Theorem 2 Let $\mathcal{T}$ be a t-norm on $\mathcal{L}_{3}$ which is a join-morphism and which satisfies $\mathcal{T}\left(D_{3}, D_{3}\right) \subseteq D_{3}$. Assume that the mappings $g:[0,1] \rightarrow[0,1]$ and $g^{\prime}:[0,1] \rightarrow[0,1]$ defined by

$$
\begin{aligned}
g\left(y_{1}\right) & =\operatorname{pr}_{2} \mathcal{T}\left((0,1,1),\left(y_{1}, y_{1}, y_{1}\right)\right), \\
g^{\prime}\left(y_{1}\right) & =\operatorname{pr}_{3} \mathcal{T}\left((0,0,1),\left(y_{1}, y_{1}, y_{1}\right)\right),
\end{aligned}
$$

for all $y_{1} \in[0,1]$, satisfy $g=g^{\prime}=\mathrm{id}_{[0,1]}$. Define $T$ as in Lemma 3 and $t_{2,1}, t_{3,1}, t_{3,2}$ and $t_{3,3}$ as in Lemma 8. Then, for all $x$ and $y$ in $L_{3}$,

$$
\begin{array}{rl}
\operatorname{pr}_{3} & \mathcal{T}(x, y) \\
=\sup \left(T\left(t_{3,1}, T\left(x_{3}, y_{3}\right)\right), T\left(t_{3,2}, T\left(x_{2}, y_{3}\right)\right),\right. \\
& T\left(t_{3,2}, T\left(x_{3}, y_{2}\right)\right), T\left(t_{3,3}, T\left(x_{2}, y_{2}\right)\right), \\
& \left.T\left(x_{1}, y_{3}\right), T\left(x_{3}, y_{1}\right)\right),
\end{array}
$$

$T$ is a join-morphism and

$$
T\left(t_{3,1}, t_{3,2}\right)=\sup \left(T\left(t_{3,1}, t_{3,3}\right), T\left(t_{3,2}, t_{2,1}\right)\right) .
$$

Proof. The representation of $\operatorname{pr}_{3} \mathcal{T}(x, y)$ and the last equality follow easily from Lemma 3, Lemma 6, Lemma 7 and Lemma 8 . The fact that $T$ is a join-morphism follows from Theorem 1.

We now obtain the main result.

Theorem $3 A$ mapping $\mathcal{T}: L_{3}^{2} \rightarrow L_{3}$ is a t-norm on $\mathcal{L}_{3}$

- which is a join-morphism,

- which satisfies $\mathcal{T}\left(D_{3}, D_{3}\right) \subseteq D_{3}$, and

- for which the mappings $g:[0,1] \rightarrow[0,1]$ and $g^{\prime}:[0,1] \rightarrow[0,1]$ defined by

$$
\begin{aligned}
g\left(y_{1}\right) & =\operatorname{pr}_{2} \mathcal{T}\left((0,1,1),\left(y_{1}, y_{1}, y_{1}\right)\right), \\
g^{\prime}\left(y_{1}\right) & =\operatorname{pr}_{3} \mathcal{T}\left((0,0,1),\left(y_{1}, y_{1}, y_{1}\right)\right),
\end{aligned}
$$

for all $y_{1} \in[0,1]$, satisfy $g=g^{\prime}=\mathrm{id}_{[0,1]}$ 
if and only if there exists a $t$-norm $T$ on $([0,1], \leq)$ which is a join-morphism and elements $t_{2,1}, t_{3,1}$, $t_{3,2}$ and $t_{3,3}$ of $[0,1]$ such that for all $x$ and $y$ in $L_{3}$,

$$
\begin{aligned}
& \mathcal{T}(x, y) \\
& =\left(T\left(x_{1}, y_{1}\right),\right. \\
& \quad \sup \left(T\left(t_{2,1}, T\left(x_{2}, y_{2}\right)\right), T\left(x_{1}, y_{2}\right), T\left(x_{2}, y_{1}\right)\right), \\
& \quad \sup \left(T\left(t_{3,1}, T\left(x_{3}, y_{3}\right)\right), T\left(t_{3,2}, T\left(x_{2}, y_{3}\right)\right),\right. \\
& \quad T\left(t_{3,2}, T\left(x_{3}, y_{2}\right)\right), T\left(t_{3,3}, T\left(x_{2}, y_{2}\right)\right), \\
& \left.\left.\quad T\left(x_{1}, y_{3}\right), T\left(x_{3}, y_{1}\right)\right)\right),
\end{aligned}
$$

and

$$
T\left(t_{3,1}, t_{3,2}\right)=\sup \left(T\left(t_{3,1}, t_{3,3}\right), T\left(t_{3,2}, t_{2,1}\right)\right) .
$$

Proof. The sufficient condition is shown in Theorem 1 and Theorem 2. We show the necessary condition. Assume that there exists a t-norm $T$ on the unit interval which is a join-morphism, let $t_{2,1}, t_{3,1}$, $t_{3,2}$ and $t_{3,3}$ be arbitrary elements of $[0,1]$ for which (4) holds and define $\mathcal{T}: L_{3}^{2} \rightarrow L_{3}$ by (3), for all $x$ and $y$ in $L_{3}$. A direct calculation shows that $\mathcal{T}$ is a join-morphism, $\mathcal{T}\left(D_{3}, D_{3}\right) \subseteq D_{3}$ and the mappings $g$ and $g^{\prime}$ satisfy $g=g^{\prime}=\mathrm{id}_{[0,1]}$. It is also straightforward to see that $\mathcal{T}$ is commutative and increasing in both arguments and that $\mathcal{T}\left(1_{\mathcal{L}_{3}}, x\right)=x$, for all $x \in L_{3}$.

We show that $\mathcal{T}$ is associative. Let $x, y$ and $z$ in $L_{3}$. From Theorem 1 it follows that $\operatorname{pr}_{1} \mathcal{T}(x$, $\mathcal{T}(y, z))=\operatorname{pr}_{1} \mathcal{T}(\mathcal{T}(x, y), z)$ and $\operatorname{pr}_{2} \mathcal{T}(x, \mathcal{T}(y, z))=$ $\operatorname{pr}_{2} \mathcal{T}(\mathcal{T}(x, y), z)$. We calculate

$$
\begin{aligned}
& \operatorname{pr}_{3} \mathcal{T}(x, \mathcal{T}(y, z)) \\
& =\sup \left(T\left(t_{3,1}, T\left(x_{3}, \operatorname{pr}_{3} \mathcal{T}(y, z)\right)\right)\right. \text {, } \\
& T\left(t_{3,2}, T\left(x_{2}, \operatorname{pr}_{3} \mathcal{T}(y, z)\right)\right), \\
& T\left(t_{3,2}, T\left(x_{3}, \operatorname{pr}_{2} \mathcal{T}(y, z)\right)\right), \\
& T\left(t_{3,3}, T\left(x_{2}, \operatorname{pr}_{2} \mathcal{T}(y, z)\right)\right), \\
& \left.T\left(x_{1}, \operatorname{pr}_{3} \mathcal{T}(y, z)\right), T\left(x_{3}, \operatorname{pr}_{1} \mathcal{T}(y, z)\right)\right) \\
& =\sup \left(T\left(t_{3,1}, T\left(x_{3}, T\left(t_{3,1}, T\left(y_{3}, z_{3}\right)\right)\right)\right)\right. \text {, } \\
& T\left(t_{3,1}, T\left(x_{2}, T\left(t_{3,2}, T\left(y_{2}, z_{3}\right)\right)\right)\right), \\
& T\left(t_{3,1}, T\left(x_{3}, T\left(t_{3,2}, T\left(y_{3}, z_{2}\right)\right)\right)\right) \text {, } \\
& T\left(t_{3,1}, T\left(x_{3}, T\left(t_{3,3}, T\left(y_{2}, z_{2}\right)\right)\right)\right), \\
& T\left(t_{3,1}, T\left(x_{3}, T\left(y_{1}, z_{3}\right)\right)\right) \text {, } \\
& T\left(t_{3,1}, T\left(x_{3}, T\left(y_{3}, z_{1}\right)\right)\right) \text {, } \\
& T\left(t_{3,2}, T\left(x_{2}, T\left(t_{3,1}, T\left(y_{3}, z_{3}\right)\right)\right)\right) \text {, } \\
& T\left(t_{3,2}, T\left(x_{2}, T\left(t_{3,2}, T\left(y_{2}, z_{3}\right)\right)\right)\right) \text {, } \\
& T\left(t_{3,2}, T\left(x_{2}, T\left(t_{3,2}, T\left(y_{3}, z_{2}\right)\right)\right)\right) \text {, } \\
& T\left(t_{3,2}, T\left(x_{2}, T\left(t_{3,3}, T\left(y_{2}, z_{2}\right)\right)\right)\right) \text {, } \\
& T\left(t_{3,2}, T\left(x_{2}, T\left(y_{1}, z_{3}\right)\right)\right) \text {, } \\
& T\left(t_{3,2}, T\left(x_{2}, T\left(y_{3}, z_{1}\right)\right)\right) \text {, } \\
& T\left(t_{3,2}, T\left(x_{3}, T\left(t_{2,1}, T\left(y_{2}, z_{2}\right)\right)\right)\right) \text {, } \\
& T\left(t_{3,2}, T\left(x_{3}, T\left(y_{1}, z_{2}\right)\right)\right) \text {, } \\
& T\left(t_{3,2}, T\left(x_{3}, T\left(y_{2}, z_{1}\right)\right)\right) \text {, } \\
& T\left(t_{3,3}, T\left(x_{2}, T\left(t_{2,1}, T\left(y_{2}, z_{2}\right)\right)\right)\right) \text {, } \\
& T\left(t_{3,3}, T\left(x_{2}, T\left(y_{1}, z_{2}\right)\right)\right) \text {, }
\end{aligned}
$$

$$
\begin{aligned}
& T\left(t_{3,3}, T\left(x_{2}, T\left(y_{2}, z_{1}\right)\right)\right), \\
& T\left(x_{1}, T\left(t_{3,1}, T\left(y_{3}, z_{3}\right)\right)\right), \\
& T\left(x_{1}, T\left(t_{3,2}, T\left(y_{2}, z_{3}\right)\right)\right), \\
& T\left(x_{1}, T\left(t_{3,2}, T\left(y_{3}, z_{2}\right)\right)\right), \\
& T\left(x_{1}, T\left(t_{3,3}, T\left(y_{2}, z_{2}\right)\right)\right), \\
& T\left(x_{1}, T\left(y_{1}, z_{3}\right)\right), \\
& T\left(x_{1}, T\left(y_{3}, z_{1}\right)\right), \\
& \left.T\left(x_{3}, T\left(y_{1}, z_{1}\right)\right)\right)
\end{aligned}
$$

Using the commutativity and associativity of $T$ we see that (6), (15) and (21) are symmetric in $x, y$ and $z,(7),(8)$ and (12) are symmetric in $x, y$ and $z,(10)$, (11) and (24) are symmetric in $x, y$ and $z,(16),(17)$, (19), (20), (25) and (26) are symmetric in $x, y$ and $z,(22),(23)$ and (27) are symmetric in $x, y$ and $z$, and (28), (29) and (30) are symmetric in $x, y$ and $z$. Furthermore, taking in consideration (4), we see that (9), (13), (14) and (18) are symmetric in $x, y$ and $z$. Hence $\operatorname{pr}_{3} \mathcal{T}(x, \mathcal{T}(y, z))=\operatorname{pr}_{3} \mathcal{T}(\mathcal{T}(x, y), z)$.

Remark 1 Let $T$ be an arbitrary t-norm on $([0,1], \leq)$ and $t_{2,1}, t_{3,1}, t_{3,2}$ and $t_{3,3}$ be arbitrary elements of $[0,1]$. Define a mapping $\mathcal{T}:\left(L_{3}\right)^{2} \rightarrow L_{3}$ by (3), for all $x$ and $y$ in $L_{3}$. Then $\mathcal{T}$ is a $t$-norm on $\mathcal{L}_{3}$ if and only if (4) holds.

Indeed, using (6)-(30) with $x=(0,1,1), y=$ $(0,1,1)$ and $z=(0,0,1)$, a direct calculation shows that from the associativity of $\mathcal{T}$ it follows that (4) holds. Conversely, if (4) holds, then a similar calculation as in the proof of Theorem 3 shows that $\mathcal{T}$ is a t-norm.

Example 1 Let $T$ be a t-norm on $([0,1], \leq)$. The smallest t-norm $\mathcal{T}$ which can be obtained using (3) is obtained for $t_{2,1}=t_{3,1}=t_{3,2}=t_{3,3}=0$ : for all $x$ and $y$ in $L_{3}$,

$$
\begin{gathered}
\mathcal{T}(x, y)=\left(T\left(x_{1}, y_{1}\right), \sup \left(T\left(x_{1}, y_{2}\right), T\left(x_{2}, y_{1}\right)\right),\right. \\
\left.\sup \left(T\left(x_{1}, y_{3}\right), T\left(x_{3}, y_{1}\right)\right)\right) .
\end{gathered}
$$

We see that the third component of the output only depends on the first and third components of the input values. This can be seen as a pessimistic extension of $T$ to 3 -dimensional fuzzy sets. Indeed, for the calculation of all three components we have to aggregate the corresponding component for one of the input values with the first component (i.e. the smallest component) of the other input value.

If we want the third component to be dependent of the second and third components of the input values instead, we must set $t_{2,1}=t_{3,1}=0$ and $t_{3,2}=t_{3,3}=1$. We obtain for all $x$ and $y$ in $L_{3}$ :

$$
\begin{gathered}
\mathcal{T}(x, y)=\left(T\left(x_{1}, y_{1}\right), \sup \left(T\left(x_{1}, y_{2}\right), T\left(x_{2}, y_{1}\right)\right),\right. \\
\left.\sup \left(T\left(x_{2}, y_{3}\right), T\left(x_{3}, y_{2}\right)\right)\right) .
\end{gathered}
$$

This is also a pessimistic extension, but less pessimistic than the previous example because to calculate each component, we now have to aggregate 
the corresponding component for one of the input values with the component located one level lower of the other input value.

If we put $t_{2,1}=t_{3,1}=t_{3,2}=0$ and $t_{3,3}=1$, we have for all $x$ and $y$ in $L_{3}$ :

$$
\begin{array}{r}
\mathcal{T}(x, y)=\left(T\left(x_{1}, y_{1}\right), \sup \left(T\left(x_{1}, y_{2}\right), T\left(x_{2}, y_{1}\right)\right),\right. \\
\left.\sup \left(T\left(x_{2}, y_{2}\right), T\left(x_{1}, y_{3}\right), T\left(x_{3}, y_{1}\right)\right)\right) .
\end{array}
$$

By putting $t_{2,1}=t_{3,1}=t_{3,2}=t_{3,3}=1$, we obtain the largest t-norm which can be obtained using (3): for all $x$ and $y$ in $L_{3}$,

$$
\mathcal{T}(x, y)=\left(T\left(x_{1}, y_{1}\right), T\left(x_{2}, y_{2}\right), T\left(x_{3}, y_{3}\right)\right) .
$$

This can be seen as an optimistic extension because for each component we obtain the highest possible value, namely the value obtained by aggregating the corresponding components of both input values.

The above notions of pessimism and optimism extend similar notions for 2-dimensional fuzzy sets described in [24].

\section{Conclusion}

In this paper we investigated triangular norms in 3-dimensional fuzzy set theory which are joinmorphisms. We have found that if we require several boundary conditions, then we obtain a representation which extends a similar representation for t-norms in 2-dimensional fuzzy set theory (intervalvalued fuzzy set theory, Atanassov's intuitionistic fuzzy set theory). In the future we would like to obtain a representation in the case that there is only one boundary condition, namely that exact elements are mapped to exact elements. We expect this representation to be even more complicated than the one for t-norms in 2-dimensional fuzzy set theory given in [17]. We would also like to extend the results in this paper to t-norms in $n$-dimensional fuzzy set theory, for $n>3$.

\section{References}

[1] L. A. Zadeh. Fuzzy sets. Information and Control, 8(3):338-353, 1965.

[2] J. A. Goguen. L-fuzzy sets. Journal of Mathematical Analysis and Applications, 18(1):145174, 1967.

[3] R. Sambuc. Fonctions $\Phi$-floues. Application à l'aide au diagnostic en pathologie thyroidienne. $\mathrm{PhD}$ thesis, Université de Marseille, France, 1975.

[4] K. T. Atanassov. Intuitionistic fuzzy sets, 1983. VII ITKR's Session, Sofia (deposed in Central Sci.-Technical Library of Bulg. Acad. of Sci., 1697/84) (in Bulgarian).

[5] K. T. Atanassov. Intuitionistic Fuzzy Sets. Physica-Verlag, Heidelberg, New York, 1999.

[6] X. S. Li, X. H. Yuan, and E. S. Lee. The three-dimensional fuzzy sets and their cut sets. Computers and Mathematics with Applications, 58(7):1349-1359, 2009.

[7] Y. G. Shang, X. H. Yuan, and E. S. Lee. The $n$-dimensional fuzzy sets and Zadeh fuzzy sets based on the finite valued fuzzy sets. Computers and Mathematics with Applications, 60(3):442-463, 2010.

[8] M. Baczyński and B. Jayaram. Fuzzy Implications, volume 231 of Studies in Fuzziness and Soft Computing. Springer, Berlin, 2008.

[9] N. Galatos, P. Jipsen, T. Kowalski, and H. Ono. Residuated Lattices: An Algebraic Glimpse at Substructural Logics, volume 151 of Studies in Logic and the Foundations of Mathematics. Elsevier, 2007.

[10] P. Hájek. Metamathematics of Fuzzy Logic. Kluwer Academic Publishers, Dordrecht, 1998.

[11] U. Höhle. Commutative, residuated l-monoids. In U. Höhle and Klement E. P., editors, Non-classical Logics and Their Applications to Fuzzy Subsets: A Handbook of the Mathematical Foundations of Fuzzy Set Theory, pages 53106. Kluwer Academic Publishers, 1995.

[12] E. P. Klement, R. Mesiar, and E. Pap. Triangular Norms. Kluwer Academic Publishers, Dordrecht, 2000.

[13] E. P. Klement, R. Mesiar, and E. Pap. Triangular norms. Position paper I: basic analytical and algebraic properties. Fuzzy Sets and Systems, 143(1):5-26, 2004.

[14] E. P. Klement, R. Mesiar, and E. Pap. Triangular norms. Position paper II: general constructions and parameterized families. Fuzzy Sets and Systems, 145(3):411-438, 2004.

[15] E. P. Klement, R. Mesiar, and E. Pap. Triangular norms. Position paper III: continuous t-norms. Fuzzy Sets and Systems, 145(3):439454, 2004.

[16] M. Baczyński. On the distributivity of implication operations over t-representable t-norms generated from strict t-norms in intervalvalued fuzzy sets theory. In E. Hüllermeier, R. Kruse, and F. Hoffmann, editors, Proceedings of the 13th International Conference on Information Processing and Management of Uncertainty in Knowledge-Based Systems (IPMU 2010), pages 637-646, Berlin, Heidelberg, 2010. Springer-Verlag.

[17] G. Deschrijver. A representation of t-norms in interval-valued $L$-fuzzy set theory. Fuzzy Sets and Systems, 159(13):1597-1618, 2008.

[18] A. Pankowska and M. Wygralak. General IFsets with triangular norms and their applications to group decision making. Information Sciences, 176(18):2713-2754, 2006.

[19] B. Van Gasse, C. Cornelis, G. Deschrijver, and E. E. Kerre. A characterization of intervalvalued residuated lattices. International Journal of Approximate Reasoning, 49(2):478-487, 2008 . 
[20] J. C. Fodor. Contrapositive symmetry of fuzzy implications. Fuzzy Sets and Systems, 69(2):141-156, 1995.

[21] E. Turunen. Mathematics Behind Fuzzy Logic. Physica-Verlag, 1999.

[22] G. Deschrijver, C. Cornelis, and E. E. Kerre. On the representation of intuitionistic fuzzy tnorms and t-conorms. IEEE Transactions on Fuzzy Systems, 12(1):45-61, 2004.

[23] G. Deschrijver and E. E. Kerre. Classes of intuitionistic fuzzy t-norms satisfying the residuation principle. International Journal of Uncertainty, Fuzziness and Knowledge-Based Systems, 11(6):691-709, 2003.

[24] G. Deschrijver and C. Cornelis. Representability in interval-valued fuzzy set theory. International Journal of Uncertainty, Fuzziness and Knowledge-Based Systems, 15(3):345-361, 2007. 